\title{
Randomised placebo controlled trial of non-invasive ventilation for hypercapnia in cystic fibrosis
}

\author{
A C Young, ${ }^{1,2}$ J W Wilson, ${ }^{1,2}$ T C Kotsimbos, ${ }^{1}$ M T Naughton ${ }^{1,2}$
}

\begin{abstract}
- Additional information on subjects, study design, interventions and outcome measures are published online only at http://thorax.bmj.com/ content/vol63/issue1

\section{${ }^{1}$ Department of Allergy Immunology and Respiratory Medicine, The Alfred Hospital, Melbourne, Victoria, Australia; ${ }^{2}$ Department of Medicine, Monash University, Melbourne, Victoria, Australia}

Correspondence to: Dr A C Young, Department of Allergy Immunology and Respiratory Medicine, The Alfred Hospital, Commercial Rd, Prahran, Melbourne, VIC, 3181, Australia; alan.young@ med.monash.edu.au

Received 11 April 2007 Accepted 2 July 2007 Published Online First 2 August 2007

\begin{abstract}
Background: The clinical benefits of domiciliary noninvasive positive pressure ventilation (NIV) have not been established in cystic fibrosis (CF). We studied the effects of nocturnal NIV on quality of life (OoL), functional and physiological outcomes in CF subjects with awake hypercapnia (arterial carbon dioxide pressure $\mathrm{PaCO}_{2}>45 \mathrm{~mm} \mathrm{Hg}$ ).
\end{abstract}

Methods: In a randomised, placebo controlled, crossover study, eight subjects with CF, mean (SD) age 37 (8) years, body mass index $21.1(2.6) \mathrm{kg} / \mathrm{m}^{2}$, forced expiratory volume in $1 \mathrm{~s} 35$ (8)\% predicted and $\mathrm{PaCO}_{2} 52$ (4) $\mathrm{mm} \mathrm{Hg}$ received 6 weeks of nocturnal (1) air (placebo), (2) oxygen and (3) NIV. The primary outcome measures were CF specific OoL, daytime sleepiness and exertional dyspnoea. Secondary outcome measures were awake and asleep gas exchange, sleep architecture, lung function and peak exercise capacity.

Results: Compared with air, NIV improved the chest symptom score in the CF OoL Questionnaire (mean difference $10 ; 95 \% \mathrm{Cl} 5$ to $16 ; \mathrm{p}=0.002$ ) and the transitional dyspnoea index score (mean difference 3.1; 95\% Cl 1.2-5.0; $p=0.01$ ). It reduced maximum nocturnal pressure of transcutaneous $\mathrm{CO}_{2}\left(\mathrm{PtcCO}_{2}\right.$ mean difference $-17 \mathrm{~mm} \mathrm{Hg} ; 95 \% \mathrm{Cl}-7$ to $-28 \mathrm{~mm} \mathrm{Hg}$; $p=0.005)$ and increased exercise performance on the Modified Shuttle Test (mean difference 83 m; 95\% Cl 21 to $144 \mathrm{~m} ; \mathrm{p}=0.02$ ). NIV did not improve sleep architecture, lung function or awake $\mathrm{PaCO}_{2}$.

Conclusion: 6 weeks of nocturnal NIV improves chest symptoms, exertional dyspnoea, nocturnal hypoventilation and peak exercise capacity in adult patients with stable CF with awake hypercapnia. Further studies are required to determine whether or not NIV can improve survival.

Cystic fibrosis (CF) is characterised by a progressive decline in lung function resulting in impaired quality of life (QoL). ${ }^{1}$ Severe lung disease impairs gas exchange and increases work of breathing while awake $e^{2}$ and particularly during sleep..$^{3-5}$ Improvements in nocturnal gas exchange and work of breathing could translate into better clinical outcomes and QoL.

Nocturnal non-invasive bi-level positive pressure ventilation (NIV) is beneficial for stable daytime hypercapnia due to kyphoscoliosis, neuromuscular disease and chronic obstructive pulmonary disease (COPD). ${ }^{6}$ Studies utilising NIV in CF have demonstrated improved gas exchange and reduced work of breathing during a single overnight session, ${ }^{3}{ }^{4}$ and improved oxygenation during physiotherapy. ${ }^{5}$ Uncontrolled non-randomised studies suggest that NIV may bridge hypercapnic CF patients to lung transplantation. ${ }^{8-10}$ Potential complications of NIV include sleep fragmentation and drying of secretions with decreased sputum clearance. A recent Cochrane review highlighted the need for randomised controlled trials to assess the clinical effects of NIV in CF. ${ }^{11}$

We hypothesised that nocturnal NIV would improve QoL, functional and physiological outcomes in subjects with stable CF with impaired nocturnal gas exchange and daytime hypercapnia. Our aim was to perform a randomised placebo controlled trial of nocturnal NIV in these "at risk" subjects with CF to assess the effects on GoL, and functional and physiological outcomes. Some of the results of these studies have been reported previously in the form of an abstract. ${ }^{12}$

\section{METHODS}

\section{Subjects}

Written informed consent was obtained after study approval by The Alfred Hospital Institutional Ethics Committee. Patients were recruited from a cross sectional study ${ }^{12}$ of 60 consecutive adult CF outpatients with mild to severe lung disease (forced expiratory volume in $1 \mathrm{~s}$ $\left(\mathrm{FEV}_{1}\right)<70 \%$ predicted) who attended The Alfred Hospital CF Service. Inclusion criteria for the current study were: $\mathrm{FEV}_{1} \leqslant 70 \%$ predicted, clinical stability (no hospitalisation or new antibiotics 2 weeks prior), positive screening overnight oximetry $\left(\mathrm{SpO}_{2}<90 \%\right.$ for $\geqslant 5 \%$ of the night), impaired gas exchange on polysomnography $\left(\mathrm{SpO}_{2}<90 \%\right.$ for $\geqslant 5 \%$ of total sleep time and/or rise in pressure of transcutaneous $\mathrm{CO}_{2}\left(\mathrm{PtcCO}_{2}\right) \geqslant 5 \mathrm{~mm} \mathrm{Hg}$ nonrapid eye movement (NREM) to rapid eye movement (REM) sleep) and awake hypercapnia $\left(\mathrm{PaCO}_{2}\right.$ $>45 \mathrm{~mm} \mathrm{Hg}$ ). Exclusion criteria were: previous domiciliary oxygen $\left(\mathrm{O}_{2}\right)$ or NIV, current sedative use, cardiac or neurological disease and obstructive sleep apnoea.

\section{Study design}

The trial design was randomised placebo controlled crossover. Each subject received 6 weeks of nocturnal (1) air (placebo), (2) $\mathrm{O}_{2}$ and (3) NIV in random order separated by a 2 week washout period. Data were collected at baseline and at the end of each treatment period (fig 1). Treatments were commenced, and post-treatment assessments were carried out during a period of clinical stability (defined as no current need for hospitalisation or intravenous antibiotics).

\section{Interventions}

NIV (BiPAP Synchrony S/T; Respironics, Pennsylvania, USA) with humidification (HC 150; Fisher and Paykel, Laguna Hills, California, USA) was delivered via nasal or full face mask fitted to 
Figure 1 Patient enrolment and study design. NIV, non-invasive positive pressure ventilation; PSG, polysomnography.

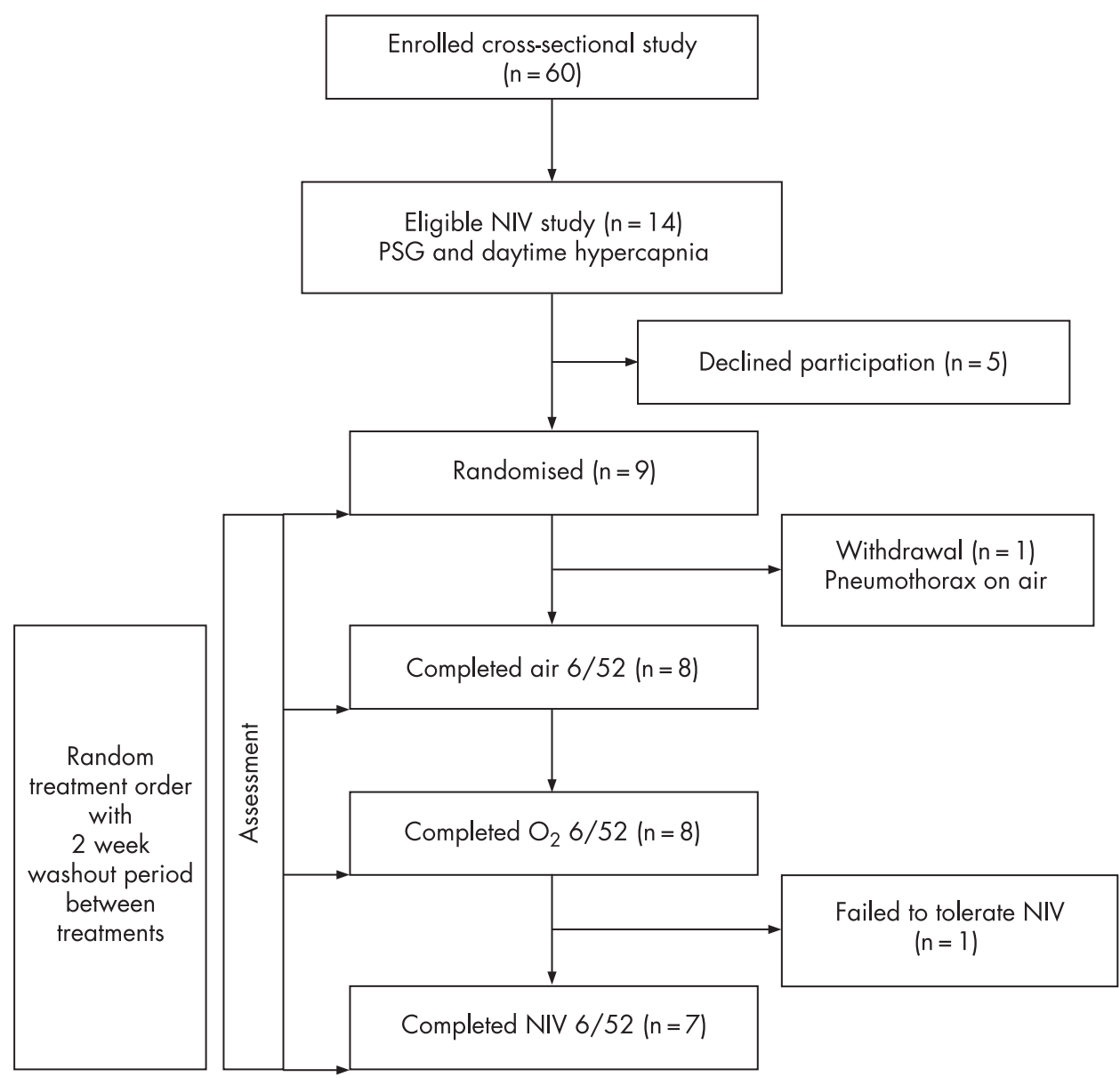

patient comfort. Acclimatisation involved an afternoon session of several hours with an experienced respiratory therapist and sleep physician to optimise mask fit and ensure patients could tolerate therapy. Polysomnography (PSG) was used to titrate NIV settings thus: inspiratory positive airway pressure (IPAP) was increased in $2 \mathrm{~cm} \mathrm{H}_{2} \mathrm{O}$ increments to attenuate any rise in $\mathrm{PtcCO}_{2} \geqslant 5 \mathrm{~mm} \mathrm{Hg}$ from NREM to REM sleep, expiratory positive airway pressure was increased in $1 \mathrm{~cm} \mathrm{H} \mathrm{H}_{2} \mathrm{O}$ increments to eliminate any obstructive events, a backup rate was added if the patient failed to trigger the ventilator. Oxygen therapy was not added to NIV as the hypothesis being tested was that NIV alone would improve outcomes.

The 6 weeks of $\mathrm{O}_{2}$ therapy was delivered from an $\mathrm{O}_{2}$ concentrator (Elite; AirSep, New York, USA) via nasal cannulae. At commencement, therapy was titrated using overnight oximetry. $\mathrm{O}_{2}$ was increased in $1 \mathrm{l} / \mathrm{min}$ increments to maintain $\mathrm{SpO}_{2}>90 \%$ for the entire night. The 6 weeks of air (placebo) was delivered through a disabled $\mathrm{O}_{2}$ concentrator and the flow rate was increased from 1 to $2 \mathrm{l}$ /min during a sham "titration" session with overnight oximetry. Thus patients remained blinded as to whether they were receiving $\mathrm{O}_{2}$ or air. NIV and concentrator hours of usage for the entire treatment period were recorded.

\section{Primary outcome measures}

Validated QoL measures assessed CF specific domains (CF OoL Questionnaire), ${ }^{13}$ sleepiness (Epworth Sleepiness Scale, Pittsburgh Sleep Quality Index ${ }^{14} 15$ and dyspnoea (Medical Research Council Dyspnoea Scale, Baseline and Transitional Dyspnoea Index). ${ }^{16}{ }^{17} \mathrm{~A}$ priori, four domains of the CF QoL were selected (physical functioning, treatment issues, chest symptoms, emotional responses) based on our clinical experience of symptoms that might be expected to improve with nocturnal NIV. The CF Subjective Sleep Disturbance Questionnaire was developed inhouse and assessed six subjective symptoms of sleep disturbance. We have previously reported an association between higher/worse scores and nocturnal hypoxia in the form of an abstract. ${ }^{12}$ Subjects were asked "which treatment was most comfortable?" and "which treatment would you prefer to continue?" at study end.

\section{Secondary outcome measures}

PSG (Compumedics E-series, Abbottsford, Australia) was manually scored, blinded to the patient's treatment, using standard criteria. Spirometry and lung volumes (Profiler and Elite Series, Medgraphics, Minnesota, USA) meeting American Thoracic Society criteria, awake supine arterial blood gases (model 865; Bayer HealthCare, New York, USA) and the validated Modified Shuttle Test ${ }^{18}$ were performed.

Neurocognitive testing was performed at the same time of day after abstinence from caffeine and alcohol. The Psychomotor Vigilance Task, ${ }^{19}$ Stroop Color and Word Test, ${ }^{20}$ Trail Making Test, ${ }^{21}$ Controlled Oral Word Association Test ${ }^{22}$ and Digit Span Test $^{23}$ were undertaken (see online data supplement for further information regarding subjects, study design, interventions and outcome measures).

\section{Statistics}

Results are presented as mean (SD). Analysis was performed using SAS V.8.2 (SAS Institute Inc, North Carolina, USA). Data 
Table 1 Subject characteristics

\begin{tabular}{|c|c|c|c|c|}
\hline & $\begin{array}{l}\text { Screened } \\
(n=60)\end{array}$ & $\begin{array}{l}\text { Randomised } \\
(\mathrm{n}=8)\end{array}$ & $\begin{array}{l}\text { Declined } \\
(\mathrm{n}=5)\end{array}$ & $\begin{array}{l}\text { Randomised } \\
\text { vs declined } \\
\text { p value }\end{array}$ \\
\hline Age (y) & $31(8)$ & $37(8)$ & $24(5)$ & 0.004 \\
\hline Sex (M:F) & $36: 24$ & $6: 2$ & $2: 3$ & \\
\hline Homozygous $\Delta \mathrm{F} 508(\%)$ & 56 & 60 & 100 & \\
\hline BMI $\left(\mathrm{kg} / \mathrm{m}^{2}\right)$ & $20.8(3.2)$ & $21.1(2.6)$ & $18.0(2.9)$ & 0.10 \\
\hline $\mathrm{FEV}_{1}(\%$ pred $)$ & $42(13)$ & $35(8)$ & $27(9)$ & 0.13 \\
\hline RV/TLC (\%) & $52(11)$ & $59(8)$ & $64(7)$ & 0.26 \\
\hline $\mathrm{pH}$ & $7.41(0.03)$ & $7.39(0.02)$ & $7.42(0.03)$ & 0.05 \\
\hline $\mathrm{PaCO}_{2}(\mathrm{~mm} \mathrm{Hg})$ & $42(5)$ & $52(4)$ & $47(4)$ & 0.05 \\
\hline $\mathrm{PaO}_{2}(\mathrm{~mm} \mathrm{Hg})$ & $75(11)$ & $63(5)$ & $60(5)$ & 0.37 \\
\hline $\mathrm{Hco}_{3}(\mathrm{mmol} / \mathrm{l})$ & $26(3)$ & $30(3)$ & $30(3)$ & 0.72 \\
\hline $\mathrm{SaO}_{2}(\%)$ & $96(3)$ & $93(3)$ & $92(2)$ & 0.34 \\
\hline
\end{tabular}

Values are mean (SD)

$\mathrm{BMI}$, body mass index; $\mathrm{FEV}_{1}$, forced expiratory volume in $1 \mathrm{~s}$; $\mathrm{PaCO}_{2}$, arterial carbon dioxide pressure; $\mathrm{PaO}_{2}$, arterial oxygen pressure; $\mathrm{RV}$, residual volume; $\mathrm{SaO}_{2}$, arterial oxygen saturation; $\mathrm{TLC}$, total lung capacity.

were analysed as difference from baseline. A repeated measures analysis of variance or Freidman two way analysis of variance assessed differences between treatments, with a two sided $p$ value of 0.05 considered significant. Where a significant difference was identified, the paired t test or Wilcoxon signed rank test was used for post hoc analysis, with a p value of 0.017 considered significant to adjust for multiple comparisons. Assuming an SD of 15 points for the physical function domain of the CF QoL, ${ }^{24}$ a sample size of 18 subjects was needed to demonstrate a 10 point improvement (clinically important difference $)^{1}$ in mean score with treatment, with a power of 0.8 and a two sided $\alpha$ of 0.05 .

\section{RESULTS}

\section{Patient characteristics}

Sixty patients were screened during the cross sectional study (lung function, overnight oximetry, arterial blood gases) of whom 14 met the entry criteria for the current study. Nine patients consented to randomisation. There was one withdrawal because of pneumothorax (while on air) and one patient did not tolerate NIV as a result of mask discomfort. Data analysis for the effects of NIV and $\mathrm{O}_{2}$ included the seven and eight patients, respectively, who completed these treatment arms (fig 1).

The group $(\mathrm{n}=8)$ mean age was 37 (8) years with a body mass index (BMI) of $21.1(2.6) \mathrm{kg} / \mathrm{m}^{2}$ and $\mathrm{FEV}_{1} 35$ (8)\% predicted. There was evidence of gas trapping (residual volume/total lung capacity ratio 59 (8)\%), chronic hypercapnia ( $\mathrm{pH} 7.39$ (0.02), $\mathrm{PaCO}_{2} 52$ (4) $\mathrm{mm} \mathrm{Hg}$ ) and moderate hypoxaemia (arterial oxygen pressure $\left(\mathrm{PaO}_{2}\right) 63$ (5) $\mathrm{mm} \mathrm{Hg}$ ). Compared with patients who were randomised, the five patients declining participation had similar $\mathrm{FEV}_{1}$ (27 (9)\% predicted), BMI (18.0 (2.9) kg/m²) and $\mathrm{PaO}_{2}(60$ (5) $\mathrm{mm} \mathrm{Hg}$ ). However, they were younger (24 (5) years) with a milder degree of chronic hypercapnia $\left(\mathrm{PaCO}_{2} 47\right.$ (4) $\left.\mathrm{mm} \mathrm{Hg}\right)$ (table 1).

\section{Treatment settings and compliance}

Air was delivered at a flow rate of $2 \mathrm{l} / \mathrm{min}$ (as per the protocol) with mean usage 7.2 (2) h/night. Oxygen was delivered at a flow rate of $1.3(0.5)$ l/min with mean usage 6.5 (3) h/night. Seven of eight patients tolerated NIV (87.5\%). Mean IPAP was 12 (1) $\mathrm{cm} \mathrm{H}_{2} \mathrm{O}$, expiratory positive airway pressure 5 (0.4) $\mathrm{cm} \mathrm{H}_{2} \mathrm{O}$ and backup rate 14 (3) breaths/min. Mean NIV usage was 4.3 (2.7) h/night.

\section{Quality of Life questionnaires}

NIV significantly increased the chest symptom score in the CF QoL (mean difference 10; 95\% CI 5 to $16 ; p=0.002$ ) and the Transition Dyspnoea Index (TDI) total score (mean difference 3.1; 95\% CI 1.2-5.0; $p=0.01$ ) compared with air. Six of seven patients who tolerated NIV increased their chest symptom score by $\geqslant 5$ points (range $5-20$ ) compared with air, and five patients

Table 2 Quality of life

\begin{tabular}{|c|c|c|c|c|}
\hline & Baseline & Air & Oxygen & NIV \\
\hline \multicolumn{5}{|l|}{ CF OoL ( $0=$ worst, $100=$ best $)$} \\
\hline Physical functioning & $69(20)$ & $68(22)$ & $64(21)$ & $67(19)$ \\
\hline Chest symptoms & $65(13)$ & $64(20)$ & $68(20)$ & $71(17) \dagger$ \\
\hline Treatment issues & $58(15)$ & $67(20)$ & $63(35)$ & $65(21)$ \\
\hline Emotional responses & $83(11)$ & $85(11)$ & $83(13)$ & $79(18)$ \\
\hline $\operatorname{CSO}(0=$ worst, $100=$ best $)$ & $42(16)$ & $56(22)$ & $50(11)$ & $58(13)$ \\
\hline ESS ( $0=$ best, $24=$ worst $)$ & $9(5)$ & $7(5)$ & $7(6)$ & $7(5)$ \\
\hline Global PSOI ( $0=$ best, $21=$ worst $)$ & $7(3)$ & $7(3)$ & $6(2)$ & $6(3)$ \\
\hline MRC $(0=$ best, $4=$ worst $)$ & $2.4(0.9)$ & $2.6(1.2)$ & $2.5(0.8)$ & $2.6(1.0)$ \\
\hline TDI $(-9=$ worst,$+9=$ best $)$ & $5.8(2.7)^{*}$ & $-1.9(2.5)$ & $-0.4(1.5)$ & $1.0(1.8) \dagger$ \\
\hline \multicolumn{5}{|c|}{$\begin{array}{l}\text { Values are mean }(\mathrm{SD}) \text {. } \\
{ }^{*} \text { Baseline Dyspnoea Index score }(0=\text { worst, } 12=\text { best). } \\
\text { †p }<0.02 \text {, mean difference from baseline (NIV vs air). } \\
\mathrm{CF} \text {, cystic fibrosis; CF QoL, Cystic Fibrosis Quality of Life questionnaire; CSQ, Cystic Fibrosis Subjective Sleep Disturbance } \\
\text { Questionnaire; ESS, Epworth Sleepiness Scale; Global PSOl, global score of the Pittsburgh Sleep Quality Index; MRC, Medical } \\
\text { Research Council Dyspnoea Scale; NIV, non-invasive positive pressure ventilation; TDI, Transition Dyspnoea Index. }\end{array}$} \\
\hline
\end{tabular}


Table 3 Sleep characteristics

\begin{tabular}{lcccccc}
\hline & Baseline & \multicolumn{1}{l}{ Air } & 0xygen & p Value & NIV & p Value $\dagger$ \\
\hline TST (min) & $337(46)$ & $331(58)$ & $328(39)$ & 0.55 & $306(26)$ & 0.22 \\
SE (\% TIB) & $86(6)$ & $82(11)$ & $82(10)$ & 0.89 & $78(9)$ & 0.38 \\
Sleep latency (min) & $20(23)$ & $16(20)$ & $18(15)$ & 0.46 & $13(13)$ & 0.84 \\
Stage 1+2 (\%TST) & $62(11)$ & $60(11)$ & $58(12)$ & 0.32 & $59(11)$ & 0.65 \\
Stage 3+4 (\%TST) & $17(9)$ & $20(7)$ & $23(10)$ & 0.2 & $19(7)$ & 0.28 \\
REM (\%TST) & $21(7)$ & $20(8)$ & $20(7)$ & 0.78 & $22(7)$ & 0.09 \\
WASO (min) & $39(27)$ & $58(42)$ & $53(34)$ & 0.5 & $77(33)$ & 0.18 \\
AHI (events/h) & $10(16)$ & $4(6)$ & $4(5)$ & 0.82 & $2(1)$ & 0.19 \\
Al (events/h) & $12(5)$ & $10(3)$ & $8(3)$ & 0.55 & $12(6)$ & 0.41 \\
PLMI (events/h) & $5(10)$ & $5(6)$ & $5(8)$ & 0.82 & $14(21)$ & 0.21
\end{tabular}

Values are mean (SD).
*Mean difference from baseline (oxygen vs air).
†Mean difference from baseline (NIV vs air).
AHI, apnoea-hypopnoea index; Al, arousal index; NIV, non-invasive positive pressure ventilation; PLMI, periodic limb movement
index; REM, rapid eye movement; SE, sleep efficiency; stage 1+2, total stage 1 and 2 sleep; stage 3+4, total stage 3 and 4 sleep;
TIB, time in bed; TST, total sleep time; WASO, wakefulness after sleep onset.

increased their TDI by $\geqslant 2$ points (range $2-9$ ). There were no significant changes in these parameters with air or supplemental $\mathrm{O}_{2}$. There were no changes with any therapeutic manoeuvre in the other QOL measures (CF Subjective Sleep Disturbance Questionnaire, Epworth Sleepiness Scale, Pittsburgh Sleep Quality Index, Medical Research Council Dyspnoea Scale) (table 2).

\section{Comfort, preference}

Four patients rated $\mathrm{O}_{2}$ the most comfortable while two rated $\mathrm{O}_{2}$ and air equally comfortable. Only one patient rated NIV as the most comfortable. Four patients preferred $\mathrm{O}_{2}$ as long term therapy while three patients preferred NIV. No patients selected air as their preferred long term therapy.

\section{Polysomnography, arterial blood gases, lung function, exercise testing}

At baseline, sleep efficiency (86 (6)\%), sleep latency (20 (23) $\mathrm{min}$ ) and distribution of sleep stages were normal (table 3). However, there was significant nocturnal hypoxia (38 (43)\% total sleep time with $\mathrm{SpO}_{2}<90 \%$ ), hypercapnia

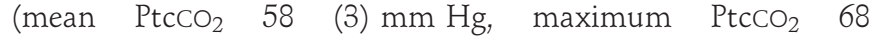
(12) $\mathrm{mm} \mathrm{Hg}$ ), tachypnoea (mean respiratory rate during slow wave sleep 23 (6) breaths/min) and tachycardia (mean heart rate 78 (15) beats/min) (table 4$)$.
Air had no significant effect on any PSG parameter, awake arterial blood gas measurements, lung function or exercise performance. Similarly, supplemental $\mathrm{O}_{2}$ had no significant effect on sleep architecture, awake arterial blood gas, lung function or exercise performance. However, during sleep there was a trend for supplemental $\mathrm{O}_{2}$ to increase mean $\mathrm{SpO}_{2}$ (mean difference $4.6 \%$; $95 \%$ CI 0.9 to $8.3 \%$; $=0.03$ ) and cause a rise in $\mathrm{PaCO}_{2}$ overnight (mean difference $4.1 \mathrm{~mm} \mathrm{Hg} ; 95 \%$ CI 0.1 to $8 \mathrm{~mm} \mathrm{Hg} ; \mathrm{p}=0.07)$, compared with air. There was no significant change in $\mathrm{PtcCO}_{2}$ with $\mathrm{O}_{2}$ therapy. NIV had no significant effect on sleep architecture, awake arterial blood gas measurements or lung function. NIV increased mean $\mathrm{SpO}_{2}$ (mean difference 3\%; 95\% CI 0.7 to $6.7 \% ; p=0.13$ ) by a similar degree as supplemental $\mathrm{O}_{2}$ but this did not reach statistical significance. NIV produced a significant reduction in maximum $\mathrm{PtcCO}_{2}$ (mean difference $-17 \mathrm{~mm} \mathrm{Hg} ; \quad 95 \%$ CI -7 to $-28 \mathrm{~mm} \mathrm{Hg} ; \mathrm{p}=0.005)$, attenuated the rise in $\mathrm{PtcCO}_{2}$ from NREM to REM sleep (mean difference $-2.4 \mathrm{~mm} \mathrm{Hg}$; 95\% CI -0.6 to $-4.2 \mathrm{~mm} \mathrm{Hg} ; \mathrm{p}=0.017$ ) and trended towards a reduction in mean nocturnal heart rate (mean difference -6.6 beats $/ \mathrm{min}$; $95 \%$ CI 0.5 to 12.6 beats $/ \mathrm{min} ; \mathrm{p}=0.05)$ compared with air. NIV also reduced maximum $\mathrm{PtcCO}_{2}$ (mean difference $-15 \mathrm{~mm} \mathrm{Hg}$; $95 \% \mathrm{CI}-4$ to $-25 \mathrm{~mm} \mathrm{Hg}$; $\mathrm{p}=0.015$ ), decreased the rise in $\mathrm{PtcCO}_{2}$ from NREM to REM sleep (mean difference $-3.1 \mathrm{~mm} \mathrm{Hg}$; $95 \%$ CI -1.2 to $-4.9 \mathrm{~mm} \mathrm{Hg}$;

Table 4 Sleep physiology

\begin{tabular}{|c|c|c|c|c|c|c|}
\hline & Baseline & Air & Oxygen & p Value* & NIV & p Value \\
\hline Mean $\mathrm{Spo}_{2}$ for TST $(\%)$ & $90(4)$ & $89(5)$ & $93(2)$ & 0.03 & $92(3)$ & 0.13 \\
\hline Minimum $\mathrm{Spo}_{2}$ for TST (\%) & $76(9)$ & $75(15)$ & $82(6)$ & 0.24 & $79(9)$ & 0.42 \\
\hline Min av $\mathrm{SpO}_{2}$ for TST (\%) & $89(4)$ & $88(6)$ & $92(1)$ & 0.03 & $91(2)$ & 0.12 \\
\hline TST with $\mathrm{SpO}_{2}<90 \%(\%)$ & $38(43)$ & $48(50)$ & $10(9)$ & 0.03 & $23(34)$ & 0.13 \\
\hline Mean $\mathrm{PtccO}_{2}$ TST (mm Hg) & $58(3)$ & $62(13)$ & $62(10)$ & 0.92 & $53(6)$ & 0.02 \\
\hline Max $\mathrm{Ptcco}_{2}$ TST (mm Hg) & $68(12)$ & $74(19)$ & $72(11)$ & 0.62 & $58(7)$ & $0.005 \%$ \\
\hline$\Delta \mathrm{PtcCO}_{2}(\mathrm{~mm} \mathrm{Hg})$ & $4.5(1.6)$ & $4.9(2.6)$ & $5.5(3.6)$ & 0.45 & $2.7(1.5)$ & $0.02 \%$ \\
\hline$\Delta \mathrm{PaCO}_{2}(\mathrm{~mm} \mathrm{Hg})$ & $1.3(3.9)$ & $2.0(2.3)$ & $6.0(4.2)$ & 0.07 & $-1.3(4.1)$ & $0.07 \$$ \\
\hline Mean RR (breaths/min) & $23(6)$ & $23(5)$ & $22(3)$ & 0.83 & $23(5)$ & 0.74 \\
\hline Mean HR (beats/min) & $78(15)$ & $73(11)$ & $70(18)$ & 0.37 & $64(15)$ & 0.05 \\
\hline
\end{tabular}

\footnotetext{
Values are mean (SD).

* Mean difference from baseline (oxygen vs air).

† Mean difference from baseline (NIV vs air).

$\$ \mathrm{p}<0.02$, mean difference from baseline (NIV vs oxygen).

$\mathrm{HR}$, heart rate; mean RR, mean respiratory rate during slow wave sleep; min av, minimum average; NIV, non-invasive positive pressure ventilation; $\mathrm{PaCO}_{2}$, arterial carbon dioxide pressure; $\Delta \mathrm{PaCO}_{2}$, change in arterial carbon dioxide pressure from evening to morning; $\mathrm{PtcCO}_{2}$, pressure of transcutaneous $\mathrm{CO}_{2} ; \Delta \mathrm{PtcCO}$, average rise in $\mathrm{PtccO}_{2}$ from non-rapid eye movement to rapid eye movement sleep; $\mathrm{SpO}_{2}$, oxygen saturation by pulse oximetry; TST, total sleep time.
} 
Table 5 Arterial blood gases, pulmonary function tests and Modified Shuttle Walk Test

\begin{tabular}{lcccc}
\hline & Baseline & Air & 0xygen & NIV \\
\hline $\mathrm{pH}$ & $7.38(0.02)$ & $7.38(0.03)$ & $7.39(0.02)$ & $7.39(0.03)$ \\
$\mathrm{PaCO}_{2}(\mathrm{~mm} \mathrm{Hg})$ & $52(4)$ & $52(7)$ & $51(7)$ & $50(5)$ \\
$\mathrm{PaO}_{2}(\mathrm{~mm} \mathrm{Hg})$ & $63(5)$ & $64(7)$ & $66(12)$ & $62(6)$ \\
$\mathrm{Hco}_{3}(\mathrm{mmol} /)$ & $30(2)$ & $30(3)$ & $30(4)$ & $30(2)$ \\
$\mathrm{SaO}_{2}(\%)$ & $93(3)$ & $93(3)$ & $94(4)$ & $92(4)$ \\
$\mathrm{FEV}_{1}(\% \mathrm{pred})$ & $35(8)$ & $32(10)$ & $32(9)$ & $33(9)$ \\
$\mathrm{FVC}(\% \mathrm{pred})$ & $60(11)$ & $54(13)$ & $54(15)$ & $58(15)$ \\
$\mathrm{RV} / \mathrm{TLC}(\%)$ & $59(8)$ & $64(9)$ & $64(11)$ & $63(10)$ \\
Shuttle Walk Test $(\mathrm{m})$ & $434(198)$ & $381(132)$ & $403(114)$ & $459(144)^{*}$ \\
\hline
\end{tabular}

Values are mean (SD).

${ }^{*} \mathrm{p}<0.02$, mean difference from baseline (NIV vs air).

$\mathrm{FEV}_{1}$, forced expiratory volume in $1 \mathrm{~s}$; FVC, forced vital capacity; NIV, non-invasive positive pressure ventilation; $\mathrm{PaCO}_{2}$, arterial carbon dioxide pressure; $\mathrm{PaO}_{2}$, arterial oxygen pressure; $\mathrm{RV}$, residual volume; $\mathrm{SaO}_{2}$, arterial oxygen saturation; TLC, total lung capacity.

$\mathrm{p}=0.006$ ) and attenuated the rise in $\mathrm{PaCO}_{2}$ overnight (mean difference $-8 \mathrm{~mm} \mathrm{Hg}$; $95 \% \mathrm{CI}-4$ to $-12 \mathrm{~mm} \mathrm{Hg}$; $=0.003$ ) compared with $\mathrm{O}_{2}$ (tables 4, 5). Furthermore, NIV produced a significant increase in shuttle distance (mean difference $83 \mathrm{~m}$; 95\% CI 21 to $144 \mathrm{~m}$; p =0.016) compared with air (table 5). Six of seven patients who tolerated NIV increased their shuttle distance by $\geqslant 50 \mathrm{~m}$ (range 50-160) compared with air.

\section{Neurocognitive testing}

There were no significant changes in neurocognitive function with any intervention (see online data supplement for further details).

\section{Adverse effects}

There were no significant side effects with air or $\mathrm{O}_{2}$ therapy. One patient developed a spontaneous pneumothorax while on air which was considered to be coincidental. Two patients complained of aerophagia with NIV which resolved with a reduction in IPAP by $2 \mathrm{~cm} \mathrm{H} \mathrm{H}_{2} \mathrm{O}$. There was no significant skin breakdown related to mask usage. No patients reported any worsening of mucus plugging or drying of secretions with NIV.

\section{DISCUSSION}

This is the first randomised placebo controlled trial of domiciliary NIV in CF subjects with hypercapnia. The results of this study indicate that 6 weeks of nocturnal NIV improves nocturnal hypoventilation, chest symptoms, exertional dyspnoea and peak exercise capacity, compared with placebo.

Our findings are consistent with published NIV data in CF on several levels. Our NIV tolerance rate of $87.5 \%$ (seven out of eight patients) was similar to the $62-83 \%$ seen in medium term observational NIV studies ${ }^{25}{ }^{26}$ in CF. Our ventilator measured compliance was $4.3 \mathrm{~h}$ per night. Attenuation of the rise in $\mathrm{PtcCO}_{2}$ with NIV and worsening of $\mathrm{PaCO}_{2}$ with $\mathrm{O}_{2}$ therapy has been reported in single overnight sessions in CF. ${ }^{3}{ }^{4} \mathrm{~A}$ meaningful improvement in dyspnoea score was reported in three of eight subjects during a 2 month trial with NIV. ${ }^{26}$ We demonstrated an increase in TDI of 3.1 units with NIV compared with placebo, which exceeds the minimally important clinical difference of 1 unit for this test. ${ }^{27}$ Five of seven subjects (71\%) improved their TDI by a margin of $\geqslant 2$ points demonstrating that the majority of subjects experienced a significant improvement.

We were unable to demonstrate a fall in awake hypercapnia with NIV, despite a sustained reduction in nocturnal $\mathrm{CO}_{2}$ levels. Uncontrolled studies of NIV in CF have shown a reduction in daytime $\mathrm{PaCO}_{2},{ }^{25}$ but results have been conflicting. ${ }^{26}$ Our results may be due to a type II error, low levels of IPAP or a short treatment period with mild baseline hypercapnia. Patients underwent rigorous in-lab titration of IPAP and checking for mask leaks. Aerophagia was seen in two patients, indicating that higher pressures were not likely to be tolerated. Our protocol duration of 5 months was chosen to maximise recruitment, minimise dropouts as a result of lung transplantation and decrease baseline fluctuations which can weaken a crossover study.

This is the first study to demonstrate an improvement in exercise performance using nocturnal NIV in CF. Compared with placebo, NIV increased mean shuttle distance by $83 \mathrm{~m}$, which exceeds the $40 \mathrm{~m}$ cut-off representative of a significant improvement in exercise performance. ${ }^{28}$ Six of seven subjects $(86 \%)$ improved their shuttle distance by $\geqslant 50 \mathrm{~m}$ which is considerable given this is an incremental test. It is important to note that some of this difference was explained by a fall in exercise capacity while on placebo, as the absolute increase in mean shuttle distance from baseline with NIV was $25 \mathrm{~m}$. A possible explanation is that NIV prevented the deterioration in exercise capacity seen with placebo. However, this hypothesis requires further study before any firm conclusions can be drawn.

Exercise performance with NIV has rarely undergone objective assessment in previous studies. Improvements in dyspnoea and exercise performance have been observed in COPD with the application of nocturnal, daytime and exercise NIV. ${ }^{29-31}$ In CF, improved muscle strength and oxygenation during chest physiotherapy has been achieved with the addition of inspiratory pressure support. ${ }^{7}$ The mechanism is likely to relate to improved gas exchange due to a reduction in the work of breathing. In CF patients with severe lung disease, NIV reduces diaphragmatic work while awake ${ }^{32} 33$ and improves alveolar ventilation while asleep. ${ }^{4}$ We postulate that unloading of the respiratory muscles overnight leads to the improved exercise capacity during the day. However, this concept requires further investigation. We found no improvement in lung function or nutritional status to account for these improvements. However, measurement of exercise flow volume loops and inspiratory capacity could further assess changes in lung mechanics. The importance of this finding cannot be underestimated as peak exercise capacity is an independent predictor of survival in both adults and children with CF. ${ }^{34} 35$

There was a strong trend towards a fall in mean nocturnal heart rate with NIV suggesting improved sympathovagal balance during sleep. A reduction in tachycardia is used 
clinically as a measure of success with NIV during acute exacerbations of COPD. Whether heart rate could be used as a marker of nocturnal work of breathing in CF remains untested.

The main strengths of this study were the inclusion of a placebo arm, random allocation of treatment order, the crossover design and objective assessment of treatment adherence. The crossover design increased the power of the study and matched baseline characteristics, which are major difficulties associated with clinical trials in CF. Nevertheless, the sample size limits further interpretation of our study and introduces the possibility of type II errors (ie, erroneously concluding no difference exists when one really does). In this context, our positive results are encouraging, given we corrected for multiple comparisons in statistical analysis to minimise type I errors (ie, erroneously concluding a difference when none exists). In addition, there was little more that could be done to increase recruitment. We are a large adult centre with 250 patients and we screened a significant proportion of our population. Most patients who declined participation cited time constraints and unwillingness to undergo multiple sleep studies and arterial blood gas measurements. This highlights the difficulty of powering studies with invasive and time consuming outcome measures in CF patients with advanced disease. Our study is of comparable size to published uncontrolled studies of NIV in CF.9 ${ }^{10} 2526$

A potential weakness of the crossover design is a change in baseline characteristics over the study period due to deteriorating health, exacerbations or carry over effects from treatment. Ideally, reassessment (eg, QoL questionnaires, lung function testing) at the start of each treatment period could have been performed but, as alluded to previously, would have been extremely onerous for patients already undertaking multiple investigations. We attempted to address these issues by ensuring clinical stability at the start and end of each treatment period and incorporating a 2 week washout period in the study design. The absence of change in lung function with any treatment argues against pulmonary exacerbations being the explanation for the observed differences. However, we cannot be certain that some of the observed changes were not due to changes in underlying disease status or carry over effects from treatment.

In conclusion, we have shown that 6 weeks of nocturnal NIV can significantly improve nocturnal hypoventilation, chest symptoms, exertional dyspnoea and peak exercise capacity in CF subjects with daytime hypercapnia. However, there is a need for larger multicentre trials to further extend these findings, clarify which subgroups may benefit most and, in particular, determine whether NIV can improve survival.

Acknowledgements: The authors would like to thank all subjects for their participation. We are also grateful to the staff of the sleep unit, cystic fibrosis unit and lung function laboratory at the Alfred Hospital for their assistance. In particular, Teanau Roebuck, Sally Ho, Seymone Doyle, Pavlina Toman, Catherine Buchan, Jo Toghill, Felicity Finlayson, Bruce Thompson and Brigitte Borg. We thank Michael Bailey and Rory Wolf for their assistance with statistical analysis.

Funding: ACY was supported by scholarships from the National Health and Medical Research Council of Australia, Monash University and The Australian Cystic Fibrosis Research Trust.

Competing interests: None.

\section{REFERENCES}

1. Gee L, Abbott J, Hart A, et al. Associations between clinical variables and quality of life in adults with cystic fibrosis. J Cyst Fibros 2005;4:59-66.

2. Hart N, Polkey Ml, Clement A, et al. Changes in pulmonary mechanics with increasing disease severity in children and young adults with cystic fibrosis. Am J Respir Crit Care Med 2002;166:61-6.
3. Gozal D. Nocturnal ventilatory support in patients with cystic fibrosis: comparison with supplemental oxygen. Eur Respir J 1997;10:1999-2003.

4. Milross MA, Piper AJ, Norman M, et al. Low-flow oxygen and bilevel ventilatory support: effects on ventilation during sleep in cystic fibrosis. Am J Respir Crit Care Med 2001;163:129-34.

5. Holland AE, Denehy L, Ntoumenopoulos G, et al. Non-invasive ventilation assists chest physiotherapy in adults with acute exacerbations of cystic fibrosis. Thorax 2003:58:880-4

6. Meecham Jones DJ, Paul EA, Jones PW, et al. Nasal pressure support ventilation plus oxygen compared with oxygen therapy alone in hypercapnic COPD. Am J Respir Crit Care Med 1995;152:538-44.

7. Fauroux B, Boule M, Lofaso $\mathrm{F}$, et al. Chest physiotherapy in cystic fibrosis: improved tolerance with nasal pressure support ventilation. Pediatrics 1999;103:E32.

8. Madden BP, Kariyawasam H, Siddiqi AJ, et al. Noninvasive ventilation in cystic fibrosis patients with acute or chronic respiratory failure. Eur Respir J 2002;19:31013.

9. Hodson ME, Madden BP, Steven MH, et al. Non-invasive mechanical ventilation for cystic fibrosis patients - a potential bridge to transplantation. Eur Respir J 1991:4:524-7.

10. Piper AJ, Parker S, Torzillo PJ, et al. Nocturnal nasal IPPV stabilizes patients with cystic fibrosis and hypercapnic respiratory failure. Chest 1992;102:846-50.

11. Moran F, Bradley J. Non-invasive ventilation for cystic fibrosis. Cochrane Database Syst Rev 2003;(2):CD002769. Chichester: Wiley InterScience, 2003.

12. Young A, Wilson J, Kotsimbos T, et al. Nocturnal oxygen desaturation is associated with decreased quality of life in cystic fibrosis. Respirology 2006;11(Suppl 2):A9.

13. Gee L, Abbott J, Conway SP, et al. Development of a disease specific health related quality of life measure for adults and adolescents with cystic fibrosis. Thorax 2000:55:946-54.

14. Buysse DJ, Reynolds CF 3rd, Monk TH, et al. The Pittsburgh Sleep Quality Index: a new instrument for psychiatric practice and research. Psychiatry Res 1989;28:193213

15. Johns MW. A new method for measuring daytime sleepiness: the Epworth sleepiness scale. Sleep 1991:14:540-5.

16. Fletcher C. Standardised questionnaire on respiratory symptoms: a statement prepared and approved by the MRC Committee on the Aetiology of Chronic Bronchitis (MRC breathlessness score). BMJ 1960;2:1665.

17. Mahler DA, Weinberg DH, Wells CK, et al. The measurement of dyspnea. Contents, interobserver agreement, and physiologic correlates of two new clinical indexes. Chest 1984:85:751-8.

18. Bradley J, Howard J, Wallace E, et al. Validity of a modified shuttle test in adult cystic fibrosis. Thorax 1999;54:437-9.

19. Dinges D, Powell J. Microcomputer analysis of performance on a portable, simple visual task RT task during sustained operations. Behav Res Methods Instrum Comput 1985:17:652-5.

20. Golden C. Stroop color and word test: A manual for clinical and experimental uses. Los Angeles: Western Psychological Services, 1978.

21. Army Individual Test Battery. Manual of directions and scoring. Washington, DC: War Department, Adjutant General's Office, 1944

22. Benton AL, Hamsher K, Sivan AB. Multilingual aphasia examination, 3rd Edn. lowa City: AJA Associates, 1983.

23. Weschler D. Weschler Adult Intelligence Scale, 3rd Edn. San Antonio: Psychological Corp, 1997.

24. Gee L, Abbott J, Conway SP, et al. Quality of life in cystic fibrosis: the impact of gender, general health perceptions and disease severity. J Cyst Fibros 2003;2:20613.

25. Hill AT, Edenborough FP, Cayton RM, et al. Long-term nasal intermittent positive pressure ventilation in patients with cystic fibrosis and hypercapnic respiratory failure (1991-1996). Respir Med 1998:92:523-6.

26. Granton JT, Shapiro C, Kesten S. Noninvasive nocturnal ventilatory support in advanced lung disease from cystic fibrosis. Respir Care 2002:47:675-81.

27. Witek TJ Jr, Mahler DA. Meaningful effect size and patterns of response of the transition dyspnea index. J Clin Epidemiol 2003;56:248-55.

28. Bradley J, Howard J, Wallace $E$, et al. Reliability, repeatability, and sensitivity of the modified shuttle test in adult cystic fibrosis. Chest 2000;117:1666-71.

29. Garrod R, Mikelsons C, Paul EA, et al. Randomized controlled trial of domiciliary noninvasive positive pressure ventilation and physical training in severe chronic obstructive pulmonary disease. Am J Respir Crit Care Med 2000;162(4 Pt 1):133541.

30. Wijkstra PJ, Lacasse $\mathrm{Y}$, Guyatt GH, et al. A meta-analysis of nocturnal noninvasive positive pressure ventilation in patients with stable COPD. Chest 2003;124:337-43.

31. Diaz 0, Begin $\mathrm{P}$, Andresen $\mathrm{M}$, et al. Physiological and clinical effects of diurnal noninvasive ventilation in hypercapnic COPD. Eur Respir J 2005:26:1016-23.

32. Fauroux $\mathbf{B}$, Pigeot $\mathrm{J}$, Polkey Ml, et al. In vivo physiologic comparison of two ventilators used for domiciliary ventilation in children with cystic fibrosis. Crit Care Med 2001;29:2097-105

33. Serra A, Polese G, Braggion C, et al. Non-invasive proportional assist and pressure support ventilation in patients with cystic fibrosis and chronic respiratory failure. Thorax 2002; 57:50-4.

34. Pianosi P, Leblanc J, Almudevar A. Peak oxygen uptake and mortality in children with cystic fibrosis. Thorax 2005;60:50-4.

35. Nixon PA, Orenstein DM, Kelsey SF, et al. The prognostic value of exercise testing in patients with cystic fibrosis. N Engl J Med 1992:327:1785-8. 